\title{
Problemi DI POLITICA EDILIZIA LOCALE NELLE ORAZIONI DI DiONE DI PRUSA
}

\section{Abstract}

\begin{abstract}
Alcune orazioni di Dione di Prusa testimoniano i temi che furono al centro della competizione politica a Prusa e in altre città della Bitinia. Tra questi, un ruolo particolare è rivestito dal programma di monumentalizzazione edilizia voluto - e in parte finanziato - da Dione per la sua città, che fu motivo (o pretesto) di attacco politico da parte dei suoi avversari. Si indagano qui i possibili riferimenti legislativi in base ai quali Dione fu accusato, tra le altre cose, di non aver portato a termine edifici promessi alla comunità o di aver raso al suolo alcune aree della città stessa per far spazio a nuovi fabbricati. L'analisi della contemporanea legislazione edilizia a Roma mostra come problemi di questo genere fossero particolarmente sentiti nel II secolo d.C., inducendo gli imperatori a legiferare in materia a più riprese.
\end{abstract}

Some speeches of Dion of Prusa attest to the themes that were at the center of the political debate in Prusa and other cities of the province of Bithynia. Dion's so-called civic speeches often refer to the building program which Dion himself proposed and partially funded with the aim of embellishing his city. However, his proposal soon became reason of political attack as Dion's political opponents accused him for not having completed the promised buildings and for having destroyed large portions of the city with the only scope of making space for his building program. The analysis of the contemporary building legislation in Rome shows how often such kind of problems affected Rome herself and other cities of the Empire, which led the emperors to frequently legislate in this regard.

1. Le orazioni di Dione di Prusa, comprese quelle cosiddette "civiche", sono state viste prevalentemente come testimonianza dell'arte retorica dell'autore e più in generale del milieu culturale a cui egli appartenne. ${ }^{1}$ Tuttavia, alcuni tra i discorsi di Dione ci restituiscono anche un quadro quanto mai vivido della vita politica delle città della Bitinia all'inizio del II secolo d.C. e dei rapporti spesso non facili che intercorrevano tra di esse. ${ }^{2}$

\footnotetext{
Seppur datato, resta fondamentale per un inquadramento della vita e dell'attività politica e culturale di Dione di Prusa von Arnim 1898, in part. pp. 308-392 per le orazioni cosiddette "bitiniche"; vd. anche Vielmetti 1941. Su Dione di Prusa, Salmeri 1980, Sheppard 1984.

2 Su cui si veda, in sintesi, Trisoglio 1972. Sulla vita politica della Bitinia al tempo di Dione di Prusa e sul coinvolgimento al suo interno di Dione stesso, si veda in particolare SALMERI 1982 e SALMERI 1999. Vd. anche Ме́тну 1994. Si veda oltre per ulteriori indicazioni bibliografiche.
} 
I discorsi dionei, ${ }^{3}$ al pari di molte delle epistole tra Traiano e Plinio il Giovane, ${ }^{4}$ richiamano alcuni tra i problemi di carattere amministrativo che maggiormente dovettero riguardare la vita pubblica delle città della Bitinia: questioni di gestione finanziaria, ${ }^{5}$ aspetti di politica edilizia, problemi relativi alle elezioni locali, accuse di appropriazione di fondi pubblici o altri beni della città sono solo alcuni dei temi che affiorano nelle orazioni di Dione.

Dione stesso fu, d'altra parte, uno dei principali attori della vita politica della sua città, a causa della vicinanza certo privilegiata che ebbe con Nerva prima e poi con Traiano, ${ }^{6} \mathrm{e}$ della sua posizione economica, che, da quanto possiamo capire dai suoi discorsi, ne faceva uno dei membri più rilevanti della locale élite cittadina. Lo troviamo infatti coinvolto in più vicende pubbliche e soprattutto troviamo riscontro di quanto Dione si spese per ottenere, anche dall'imperatore, favori di vario genere per la sua città. Contemporaneamente, però, notiamo come non pochi fossero i casi in cui egli si trovò a essere accusato da parte dei suoi concittadini, soprattutto (ma non esclusivamente) in relazione al programma edilizio di abbellimento e monumentalizzazione della città di Prusa, che Dione sostenne con forza e a più riprese e in parte finanziò.

Proprio i problemi sorti entro questo programma, che furono di natura sia edilizia che finanziaria, furono causa di accuse varie per Dione: se infatti, come è noto, atti di evergetismo edilizio privato (più o meno spontanei) risposero spesso a esigenze di autopromozione di membri delle élites locali che cercavano anche in questo modo di migliorare la propria posizione entro la compagine sociale di appartenenza, ${ }^{7}$ le orazioni di Dione ci mostrano altrettanto chiaramente come questi stessi atti potessero essere usati come pretesti di accusa contro gli evergeti stessi: ${ }^{8}$ un'opera promessa ma

\footnotetext{
In sintesi, sul gruppo di orazioni civiche, BEKKER - NIELSEN 2008, pp. 177-179.

4 L'operato di Plinio in qualità di legato in Bitinia si inserisce nella politica di Traiano volta a rafforzare il controllo sulle amministrazioni locali in sofferenza. Il caso della Bitinia, su cui torna ampiamente anche Dione di Prusa, appare particolarmente emblematico delle situazioni di difficoltà amministrativa in cui versavano le città della provincia nel II secolo d.C. Si veda a questo proposito WINTER 1996, pp. 205-207, Daguet - GAGey 2010, pp. 264-270.

5 La cattiva gestione dei beni pubblici da parte dei magistrati e delle assemblee locali, spesso a seguito di episodi di corruzione, appare come una delle cause maggiori di dissesto finanziario per le casse delle città della Bitinia (e dell'impero in generale) e come tale torna di frequente nel carteggio tra Plinio e Traiano. Nell'epistola 17, su cui si veda anche oltre, Plinio riferisce di dissesti finanziari che affliggevano Prusa e a cui erano legate le condotte di alcuni privati, che si erano appropriati di denaro pubblico destinato all'edilizia pubblica. In tema di donazioni, Plinio denuncia casi di città italiche che dilapidavano i fondi che ricevevano, confermando la diffusione di cattive gestioni amministrative (Plin. Ep. 7.18). Su questi e altri temi analoghi nel carteggio tra Plinio e Traiano, vd. Daguet-GageY 2010, Zuiderhoek 2009, pp. 42-43. Sull'attività edilizia pubblica, finanziata sia da privati che dalla cassa pubblica delle città, in Asia Minore, vd. Pont 2010, pp. 363-376 e p. 422 sulle strategie di controllo assunte dai governatori e dagli imperatori su questo particolare aspetto della vita pubblica locale. 6 Su questo aspetto si veda KoKkinia 2006. Sul rapporto con Traiano: Schmitz 1996, Амато 2014, pp. 97-118. 7 Sulla pratica dell'evergetismo edilizio esiste un'amplissima bibliografia, che tratta i molteplici aspetti che questo fenomeno assunse nel mondo romano. Qui ci limitiamo a rimandare a ENGFER 2017, pp. 76-248 con ampie indicazioni bibliografiche.

8 Sull'evergetismo di Dione di Prusa e dei suoi concittadini, pure richiamato in vario modo nei suoi discorsi, vd. KLEIJWEgt 1994.
} 
non conclusa, ad esempio, o irregolarità compiute nel processo di costruzione diventano motivi precisi e circostanziati di accusa rivolti a Dione dai suoi rivali nell'agone politico, che trovano proprio nell'ambizioso programma edilizio voluto da Dione i motivi per gettare ombre sul suo operato.

Infine, tali accuse, oltre a testimoniare i binari su cui si muoveva la competizione politica locale, riferiscono indirettamente delle leggi e delle regole che Dione era accusato di infrangere e non rispettare nell'attuazione del suo programma edilizio: evidentemente, gli avversari politici di Dione ritenevano conveniente ai propri scopi accusare l'oratore in base a riferimenti legislativi, la cui portata e il cui significato possiamo indovinare dalle parole di Dione stesso e da sparsi riscontri che troviamo ancora nell'epistolario pliniano e in fonti giuridiche.

L'immagine che emerge dalle parole di Dione, dunque, risulta particolarmente viva, composita e ricca di riferimenti storico-giuridici che, sebbene siano espressi in modi non sempre chiari, suggeriscono non solo il contesto politico ma anche il quadro giuridico entro cui si mossero Dione e i suoi concittadini. ${ }^{9}$

2. Il programma edilizio voluto, finanziato (parzialmente) e fatto approvare all'assemblea cittadina ${ }^{10}$ da Dione di Prusa aveva lo scopo dichiarato di non far sfigurare Prusa rispetto ad altre città limitrofe, con cui esistevano motivi di attrito e concorrenza. ${ }^{11}$ Tale programma, con tutti i problemi che causò a Dione, emerge in diverse delle orazioni dionee.

Un primo riferimento ricorre in 40.3-4 nel più ampio discorso che Dione pronunciò per perorare, presso i suoi concittadini, la causa di Apamea (di cui egli aveva peraltro ricevuto la cittadinanza) entro una disputa che, verosimilmente, doveva avere a che fare con diritti di proprietà terriere. ${ }^{12}$ Dione, per dimostrare la sua totale imparzialità e anzi la vicinanza a Prusa, ha modo di richiamare una sua qualche promessa, onerosa dal punto di vista finanziario ma spontanea, fatta alla sua città, facilmente identificabile con quel programma di monumentalizzazione di Prusa su cui avrà modo di tornare in questo e altri discorsi. Una promessa che, stando a quanto egli stesso afferma, tardava ad avverarsi, assumendo quindi essa, dal punto di vista del benefattore, il peso quasi di un obbligo.

È da porre l'accento sulle difficoltà, riscontrate da Dione, nell'attuazione di quanto promesso: è questo infatti un tema che non solo tornerà in altri discorsi, ma sarà anzi

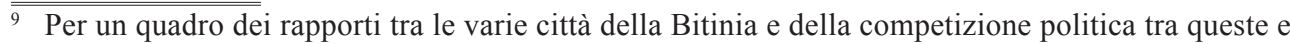
al loro interno, si veda in sintesi Heller 2006, BEKKER - NIELSEN 2008, in part. pp. 119-145. Su alcuni aspetti specifici della vita di Dione di Prusa, Amato 2014 e da ultimo Desideri 2019.

10 Per il ruolo delle assemblee cittadine nella vita politiche della Bitinia e più in generale dell'Asia Minore si veda LewIN 1995, pp. 24-42 e in generale Fernoux 2011.

11 Drolet 2004.

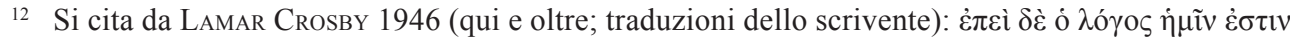

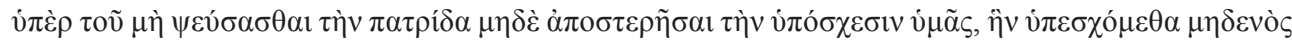

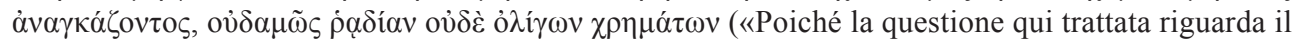
fatto che io non ho ingannato la città né le sottraggo quanto ho voi promesso (senza che nessuno mi costringesse), una promessa in nessun modo facile e che riguarda non poco denaro»).
} 
al centro delle accuse di cattiva gestione finanziaria che gli verranno rivolte. Poco oltre (40.6) ${ }^{13}$ Dione pone in risalto come la sua proposta fosse stata ripetutamente approvata dall'assemblea di Prusa e sottolinea la cura, certo gravosa, che egli ha messo nel progettare gli edifici (una stoà con altri fabbricati annessi): tale cura sarebbe stata testimoniata ${ }^{14}$ dalle misurazioni e da altre operazioni tecniche che egli aveva fatto compiere o compiuto in prima persona (pare addirittura che lui stesso fosse andato sui monti circostanti per scegliere i materiali), in modo che il progetto non risultasse inutile o inadatto, come accade - denuncia Dione - in molte altre città, dove le opere pubbliche finiscono in rovina perché non progettate in modo appropriato. Si tratta di una notazione molto importante: Dione stesso, e Plinio nelle sue epistole come vedremo, torna più volte sul problema degli errori di progettazione, che hanno fatto sì che le città della Bitinia fossero letteralmente disseminate di opere incompiute e dunque inutili.

Al paragrafo 40.8, Dione menziona per la prima volta le accuse che, a proposito delle sue iniziative edilizie (che, evidentemente, avevano subito dei ritardi), gli venivano rivolte: alcuni, non molti in verità (a suo dire), gli rinfacciano di smantellare la città, di distruggere tutto senza lasciare nulla in piedi. ${ }^{15} \mathrm{Si}$ tratta, tuttavia, della distruzione di edifici di poco conto, già in rovina, che anzi facevano vergognare $(40.9),{ }^{16}$ come Dione mette in risalto, accostandoli, ironicamente, a monumenti di grande fama come i Propilei o il Partenone di Atene. Peraltro, sembra desumersi dalle parole dionee che, prima dell'avvio della distruzione di questi edifici in rovina, il proconsole doveva aver fatto un qualche sopralluogo nell'area. Oltretutto, con grande lungimiranza, Dione ci informa che quanto aveva fatto distruggere (ed in particolare le botteghe e le officine) sarebbe stato fatto ricostruire in altro luogo (40.9). Insomma, Dione, per far spazio alle opere che aveva con grande cura progettato (e di cui avrebbe sostenuto i costi, insieme ad altri concittadini, per cui si veda oltre), aveva fatto smantellare alcuni fabbricati in rovina, e per questo, in maniera strumentale, era stato accusato di distruggere le tracce del felice passato della città. Infine, Dione ha modo di spiegare il perché del suo progetto (40.10-11): una città, come Prusa, che è stata in vario modo

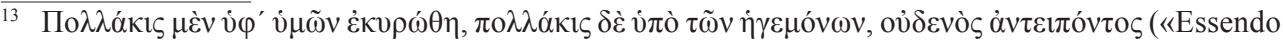
la proposta ripetutamente approvata da voi e dai proconsoli, senza che nessuno dissentisse»).

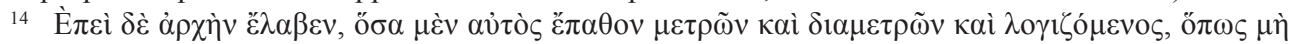

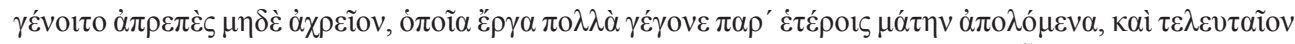

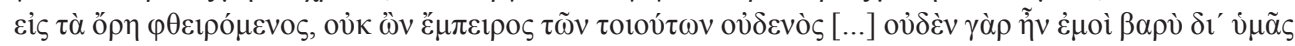

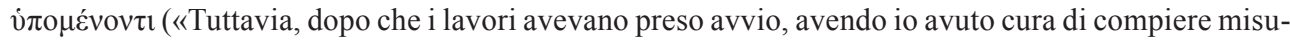
razioni, destinare gli spazi e fare calcoli, per assicurare che il progetto non risultasse inadatto né inutile, come accade con molti progetti in altre città per mancanza di progettazione, infine rovinandomi io stesso sulle montagne, pur non avendo alcuna esperienza in tali questioni»).

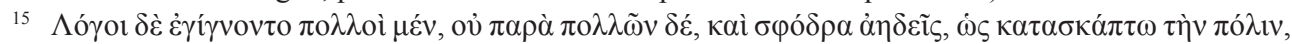

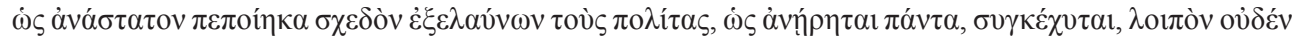
$\dot{\varepsilon} \sigma \tau \imath v$ ( «Furono fatti molti e malevoli discorsi, in realtà da parte di non molte persone, secondo i quali io sto smantellando la città, che l'ho messa a soqquadro, cacciandone infine gli abitanti, che tutto è stato distrutto e che non ne è rimasto nulla»).

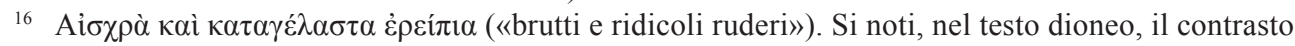
molto forte tra la grandezza degli edifici prima citati (i Propilei, il Partenone, etc.) e la pochezza dei ruderi di Prusa, della cui demolizione, peraltro a fin di bene, Dione viene accusato.
} 
onorata dall'imperatore, ad esempio divenendo sede in cui si amministrava la giustizia, necessita di un apparato monumentale che sia degno del suo status, anche in considerazione del fatto che i cittadini di molte città vicine stanno applicando programmi di abbellimento urbano analoghi. ${ }^{17}$

L'orazione quarantacinquesima ci mostra con maggiori dettagli i termini di quanto intrapreso da Dione per l'edilizia della sua città e quali fossero le accuse che i suoi avversari, riferendosi proprio a questo programma, gli rivolgevano.

Non sono chiare le circostanze in cui tale discorso, tenuto secondo von Arnim ${ }^{18}$ tra il 101 e il 102 d.C., fu pronunciato da Dione di fronte all'assemblea della sua città. Tra le altre cose, esso ribadisce, una volta in più, la generosità di Dione nei confronti di Prusa e, per l'inverso, l'ingratitudine nei suoi confronti da parte dei suoi concittadini.

Dopo un breve accenno a non meglio specificati benefici che egli avrebbe ottenuto da Traiano per la sua città (45.3-4) in contrasto ad altri cittadini di Prusa che avrebbero solo fatto finta di trattare con i proconsoli della provincia per ottenere favori per la città stessa, Dione, sempre per replicare ai suoi detrattori, ci fa indirettamente sapere che anche i governatori della provincia, in passato, avevano manifestato interesse e perplessità per l'amministrazione finanziaria della città, in particolare in relazione a un non meglio specificato progetto, forse proprio quello edilizio voluto da Dione, che rischiava di non essere completato. Tale progetto è richiamato in termini in realtà assai vaghi (45.12): ${ }^{19}$ Dione avrebbe voluto abbellire la città non solo con fontane e colonnati, ma anche con strutture più pratiche e utili come fortificazioni, attracchi e così via. E tuttavia, si lamenta, è stato accusato di voler danneggiare la città stessa. Di fronte a tali accuse, Dione ricorda ai suoi uditori che il progetto fu approvato (anche dal punto di vista finanziario) dall'assemblea nella sua totalità, senza che alcuno sollevasse obiezioni $(45.16) .{ }^{20}$

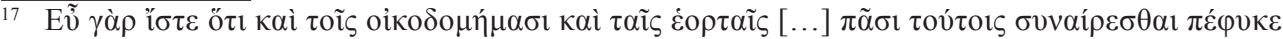

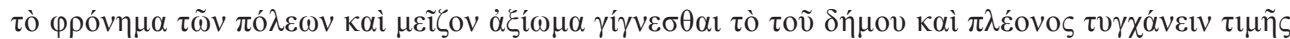

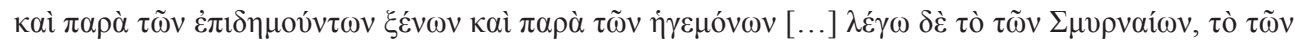

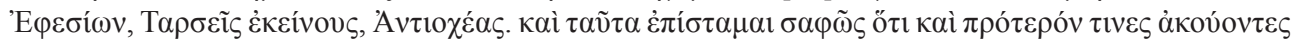

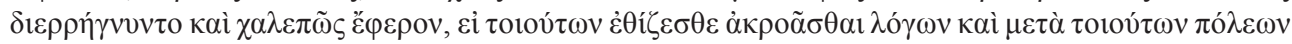

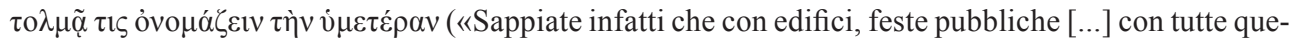
ste cose è naturale inorgoglire le città ed innalzare la dignità del popolo, e perciò ricevere grandi onori dagli stranieri come dai governatori $[. .$.$] mi riferisco alle attività dei cittadini di Efeso, a quelli di$ Tarso e agli Antiocheni»).

18 Si veda von ARNim 1898, 308-392.

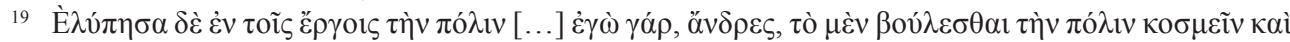

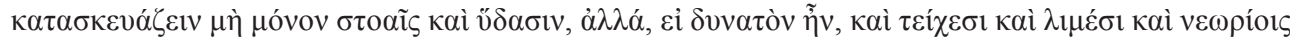
$\dot{o} \mu \mathrm{o} \lambda \mathrm{\gamma} \tilde{\omega}$ («Ho recato offesa alla città per quanto riguarda le opere pubbliche [...] Cittadini, ammetto di aver voluto abbellire e fornire la città non solo con colonnati e fontane, ma anche, se fosse possibile, con fortificazioni, porti e arsenali»).

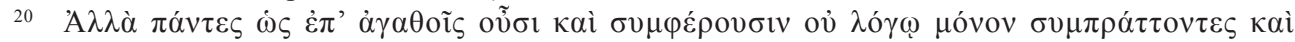

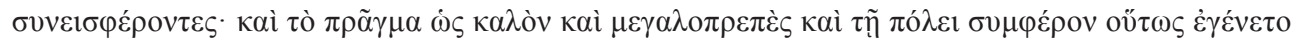
( «Al contrario tutti, pensando che [la proposta] fosse per il bene della città, non solo votando a favore, ma anche contribuendovi; e così la questione fu approvata, in quanto positiva, magnifica e di grande utilità per la città»).
} 
Le medesime accuse emergono dall'orazione quarantottesima, ${ }^{21}$ rivolte, questa volta, non solo a Dione ma anche ad altri membri dell'élite locale. Ancora una volta, ci si lamenta del ritardo relativo al completamento delle opere volute da Dione, imputato, tra le altre cose, al ritardato pagamento di somme promesse da altri cittadini di Prusa (48.11). ${ }^{22}$

In più, Dione, nell'esortazione ai suoi concittadini di non denunciare i propri affari (o malaffari) interni al proconsole Vareno in visita in città, ci fa sapere che alcuni membri dell'élite erano accusati di essere in possesso - talvolta in buona fede, talvolta in cattiva fede - di fondi pubblici (48.9). ${ }^{23}$ Le accuse non sono ulteriormente circostanziate, ma, con ogni verosimiglianza, deve trattarsi di fondi destinati a costruzioni di edifici pubblici non impiegati e trattenuti illegalmente da privati cittadini, cui erano stati affidati.

Le accuse rivolte a Dione dai suoi concittadini, così come emergono dalle orazioni, non appaiono del tutto pretestuose, ma sembrano anzi riferirsi a situazioni reali. In particolare, e proprio in relazione alla Bitinia, Plinio il Giovane, legato imperiale in provincia proprio sotto Traiano, richiama episodi del tutto simili alle vicende che coinvolsero Dione a Prusa. ${ }^{24}$

Il carteggio tra il princeps e Plinio denuncia ripetutamente la cattiva gestione finanziaria che Plinio riscontrò in molte città della provincia, spesso accompagnata a politiche edilizie poco assennate e accorte. ${ }^{25}$

$\overline{21}$ Riferimenti reiterati al programma edilizio voluto da Dione, all'appoggio ottenuto dal princeps e dai proconsoli e, per contro, all'opposizione incontrata da quest'ultimo nel tentativo di portarlo avanti emergono anche nell'orazione quarantasettesima, incentrata anch'essa sul tema della generosità (mal ripagata) di Dione nei confronti della sua città.

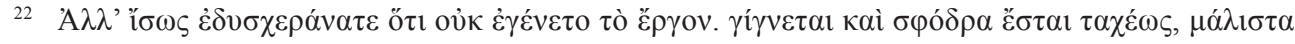

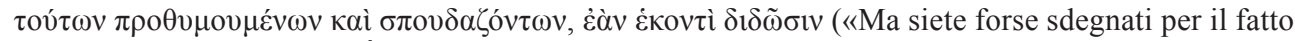
che l'opera non è conclusa. È in fase di compimento e verrà terminata rapidamente, soprattutto poiché questi sono ben disposti e si danno da fare, sempre che donino di buon grado»).

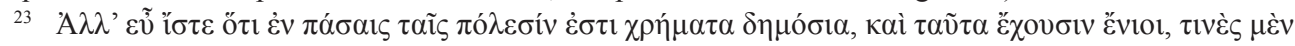

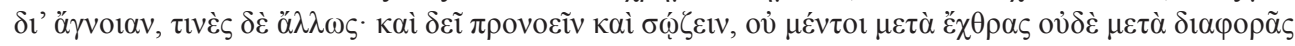
(«Ma voi sapete bene che in tutte le città ci sono fondi pubblici e che alcuni ne sono in possesso, alcuni in maniera inconsapevole, altri diversamente. Ed è necessario provvedere e recuperarli, e tuttavia non con rancore o inimicizia»).

24 Dione di Prusa appare anche in un'accusa richiamata da Plinio il Giovane nel suo carteggio con Traiano (Plin., Ep. 10.81-82), peraltro ancora una volta a proposito di questioni edilizie: Dione, che aveva fatto costruire un edificio a sue spese per la città, si rifiutava, nonostante le sollecitazioni ricevute, di presentare un rendiconto dell'opera. Gli veniva infatti imputato di aver eseguito l'opera in modo difforme rispetto a quanto progettato. Come si vede, anche in questo caso si tratta di problematiche analoghe a quelle riprese nelle orazioni dionee. Sul rapporto tra Flavio Archippo (accusatore di Dione) e Dione, certamente avversari politici, si veda Desideri 1978, p. 2, KoKkinia 2004, Billault 2014. 25 La cattiva gestione dei beni pubblici da parte dei magistrati e delle assemblee locali appare come una delle cause maggiori di dissesto finanziario per le casse delle città dell'impero, come ci mostra chiaramente il carteggio tra Plinio e Traiano. Nell'epistola 17, Plinio pone l'accento sui problemi economici della città di Prusa, a cui non sono estranee le condotte di alcuni privati, che si sono appropriati di denaro pubblico, mentre altri fondi della città sono stati spesi per motivi non chiari. Notiamo peraltro, e in relazione alle donazioni, che Plinio denuncia casi di città italiche che dilapidavano i fon- 
Proprio in relazione a Prusa, Plinio, preso atto della situazione problematica dei conti pubblici della città, caratterizzata da sprechi vari, chiede all'imperatore di inviare nella provincia dei commissari alle opere pubbliche (curatores operum) perché procedano a fare dei controlli (mensurae, nel caso specifico) e possano coì recuperare somme che Plinio definisce non mediocres (10.17b).

Problemi edilizi e relativa gestione finanziaria non oculata emergono, ancora per Prusa, in un'altra epistola pliniana (10.23): a fronte del problema, posto da Plinio a Traiano, di reperire i fondi per completare dei balnea, iniziati ma, come spesso accadeva, non completati, il princeps suggerisce a Plinio di ammonire gli abitanti di Prusa, perché in futuro si approvvigionino del denaro necessario prima di intraprendere dei lavori.

Ancora più generalizzata è la situazione denunciata in Ep. 10.39, a dimostrazione di come cattive pratiche edilizie fossero ben diffuse: nell'epistola Plinio denuncia i casi di Nicea e Claudiopoli, dove sono state spese ingenti somme per opere che, oltre a essere in taluni casi di scarsa utilità, non sono state nemmeno concluse, o per errori di progettazioni o, quel che è peggio, per l'esaurimento dei fondi a disposizione. Traiano risponde a Plinio ribadendo la necessità che i privati portino a conclusione quelle opere che avevano promesso (Ep. 10.40).

Nell'epistola 37, Plinio denuncia una situazione di dissesto finanziario della città di Nicomedia a causa di una serie di opere iniziate e non concluse, tra cui un acquedotto. Traiano, oltre a riaffermare la necessità di portare a termine l'opera, esorta Plinio ad appurare per colpa di chi si è fatto perdere tanto denaro ai Nicomediesi e si sia iniziato e non terminato l'acquedotto, verificando nel contempo se ci siano state delle ruberie a danno della cassa pubblica. In questo caso si denuncia non solo la cattiva gestione ma anche una vera e propria corruzione, menzionata anche nel discorso quarantottesimo di Dione di Prusa.

Tenendo conto anche della contiguità temporale che caratterizzò la presenza di Plinio e Dione in Bitinia, vediamo bene come le accuse che furono rivolte a Dione di non aver concluso le opere promesse e di aver gestito male il denaro occorrente, e quelle, richiamate da Dione in relazione ad altri cittadini di Prusa, di non aver onorato le promesse di donazioni o di aver sottratto alla cassa pubblica denaro destinato all'edilizia cittadina vengano fedelmente rispecchiate dall'epistolario pliniano, a dimostrazione dell'esistenza oggettiva di un problema di gestione entro la politica edilizia locale.

3. Dunque, il programma di abbellimento edilizio voluto da Dione divenne oggetto di disputa politica, dando numerosi spunti di accusa agli oppositori di Dione.

Quale che fosse la situazione politica di Prusa e al di là della concretezza di tali attacchi, qui interessa un dato in particolare, vale a dire l'orizzonte legislativo cui tali accuse dovettero rifarsi. I riferimenti a questo proposito sono, nelle orazioni dionee, praticamente mancanti. Ad esempio, quando Dione afferma di essere stato accusato di distruggere la città (per far spazio al suo progetto), non è chiaro se questa accusa fos-

$\overline{\overline{\text { di che ricevevano, }}}$ confermando la diffusione di cattive gestioni amministrative (cfr. Plin., Ep. 7.18). 
se latamente "ideologica" (oltre che pretestuosa) o se piuttosto fosse stata mossa sulla scorta di un preciso provvedimento che sanzionasse coloro che si fossero trovati in circostanze analoghe a quelle in cui si trovava Dione. Ancora, la medesima domanda si pone per l'accusa di non aver completato i lavori promessi. Come abbiamo visto sopra, in casi analoghi Plinio chiese consiglio a Traiano sull'atteggiamento da assumere nei confronti di coloro che tardavano a pagare le somme promesse, utili per portare a termine opere di utilità pubblica non ancora completate. Ci possiamo allora chiedere se esistesse una sanzione per coloro che non completassero le opere promesse.

Come ampiamente noto, la legislazione romana ebbe cura di regolamentare, sotto molteplici punti di vista, l'attività edilizia sia nell'urbs di Roma che nei centri locali dell'Italia e delle province.

In particolare, tra i vari provvedimenti che furono presi nel corso degli anni entro questo specifico settore, osserviamo la volontà, reiterata, di proteggere gli edifici da interventi non autorizzati atti alla loro demolizione parziale o totale, ${ }^{26}$ in modo da evitare operazioni di speculazione volte di solito al reimpiego o al commercio del materiale edilizio ottenuto da demolizioni; ${ }^{27}$ non secondario, per questo tipo di provvedimenti, fu l'intento di preservare il publicum decus della città. Tale attenzione emerge, non solo in due senatus consulta di età imperiale (rispettivamente del 47 d.C. e del 56 d.C.), noti da un'iscrizione proveniente da Ercolano (CIL X, 1401) ${ }^{28}$ ma anche in due leggi locali d'età repubblicana, l'una costitutiva del municipio tarentino $\left(C I L \mathrm{I}^{2}, 590 ;\right.$ ILS 6086) ${ }^{29}$ l'altra fondante della colonia cesariana di Urso in Baetica $\left(C I L \mathrm{II}^{2}, 5,1022\right) .{ }^{30}$

A Taranto, la locale legge si esprime in questi termini (11. 32-38): Nei quis in oppido quod eius municipi e[r]it aedificium detegito neiue dem [olito] / neiue disturbato, nisei quod non deterius restituturus erit, nisei d[e] s(enatus) s(ententia). I sei quis aduersus ea faxit, quant [i] id aedificium $<f>$ [u]erit, tantam pequni[a]m / municipio dare damnas esto eiusque pequniae [qu]ei uolet petiti[o] esto. / magi(stratus) quei exegerit dimidium in [p]ublicum referto, dimidium in l[u]deis, quos / publice in eo magistratu facie[t], consumito, seiue ad monumentum suom / in publico consumere uolet, l[icet]

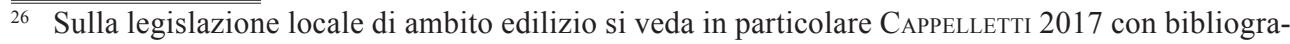
fia specifica sulla documentazione analizzata. Per Roma la bibliografia è molto vasta ed è sintetizzata in ibidem, e qui rimanderemo, per una visione generale, a PhILIPS 1973, RAINER 1987, SARGENTI 1983, SARgenti 1984, ZaCCARIA Ruggiu 1990. In età imperiale, si ribadì il divieto di demolire gli edifici, a Roma e in Italia, per trarne materiale da costruzione: Procchi 2001a, Procchi 2001b, Buongiorno 2010a. Sulla protezione degli edifici a Roma e il concetto di publicum decus, Thomas 1998, Dubouloz 2011, pp. 66-79, in part. p. 68 per i provvedimenti, di analogo significato, di età adrianea e severiana (su cui si veda anche oltre).

27 Un caso noto è quello del censore Q. Fulvio Flacco che, con grande scandalo, fece parzialmente scoperchiare nel 173 a.C. il tempio di Giunione Lacinia per riutilizzarne le tegole a Roma (Liv. 42.3.1-6). 28 Si veda Buongionno 2010b per le importantissime testimonianze in tema di politica edilizia e annessi divieti di speculazione contenute nel senatus consultum "osidiano" e nel senatus consultum "volusiano".

29 Per lo statuto tarantino si veda in particolare CAPPELletti 2011.

30 Per la Lex Ursonensis si veda in generale CRAWFORD 1996, I, pp. 393-454 n. 25, e l'aggiornamento di Caballos Rufino 2018.
} 
o idque ei s(ine) $f$ (raude) $s$ (ua) facere liceto. La regola prevista disponeva il divieto di scoperchiare, demolire o distruggere un edificio situato nel municipio e nel suo territorio. Tuttavia, il divieto decadeva se l'intervento si fosse tradotto in una miglioria concreta dell' edificio o nel caso in cui il locale senato si fosse espresso in suo favore. ${ }^{31}$

A Urso, è il capitolo 75 della Lex Coloniae Genetiuae Iuliae a regolare la materia: Ne quis in oppido coloniae Iuliae aedificium detegito neue demolito neue disturbato, nisi si praedes Inirum arbitratu dederit se redaedificaturum, aut nisi decuriones decreuerint, dum ne minus L adsint, cum ea res consulatur. Si quis aduersus ea fecerit, quanti ea res erit, tantam pecuniam colonis coloniae Genetiuae Iuliae dare damnas esto, eiusque pecuniae qui uolet petitio persecutioque ex hac lege esto. Quanto previsto a Urso richiama molto da vicino le disposizioni tarantine, sebbene manchi il riferimento all'organizzazione di ludi grazie al denaro proveniente dalla sanzione applicata in caso di mancato rispetto della regola (per contro, a Urso è richiesta una maggioranza qualificata per l'approvazione di richieste di demolizione o altre operazioni a carico di edifici). Ancora una volta ci troviamo di fronte a disposizioni che, pur con varianti non solo formali, regolavano attività di distruzione o scoperchiamento di edifici e successiva opera di ricostruzione. ${ }^{32}$

Analoghi provvedimenti furono successivamente inseriti nella Lex Flavia municipalis. Il capitolo 62 della Lex Irnitana stabilisce che nessuno possa scoperchiare, distruggere o demolire edifici entro le mura (o a esse adiacenti), se non per decisione della maggioranza dei decurioni e solo a condizione di ricostruire il fabbricato entro l'anno successivo. Come a Urso, anche in questo caso è previsto per colui che contravvenga alle regole il pagamento di una multa ai municipes di Irni..$^{33}$

Con analogie (si veda ad esempio la necessità di far decidere in materia di politica edilizia a una maggioranza qualificante dei decurioni) e differenze (l'importo della multa, la modalità tramite cui essa era comminata, o ancora gli uso previsti per il denaro così raccolto), gli statuti locali sopra richiamati sono accomunati dalla costante necessità di proteggere il patrimonio edilizio cittadino da speculazioni e soprattutto da demolizioni non autorizzate o che non prevedessero, in particolare, la riedificazione del medesimo edificio. Resta, in estrema sintesi, costante la predisposizione di divieti relativi ad operazioni di demolizione di edifici preesistenti che non fosse motivata da un loro restauro. ${ }^{34}$

Laddove nel senatus consultum "osidiano" la demolizione di edifici è espressamente proibita se intesa ad alimentare l'altrimenti lucroso commercio di materiali edilizi, ${ }^{35}$

\footnotetext{
$\overline{31}$ Su queste linee dello statuto tarantino si veda in particolare LAFFI 2007, pp. 217-219. Si veda anche Gabba 1994, pp. 110-116, Rainer 1987, pp. 284-287, Rainer 1991, CAPpelletti 2011, pp. 160-170.

32 GabBa 1994, pp. 111-113.

33 Per la Lex Irnitana si veda in particolare GonZÁLEZ, CRAWFORD 1986 e l'ampio commento di LAMBERTI 1993.

34 Su questa pratica e la legislazione ad essa riferibile, vd. in sintesi e con indicazioni bibliografiche MARANO 2013.

35 Buongionno 2010b, p. 239, che riferisce anche di un senatus consultum dell'età di Vespasiano (citato da una costituzione di Alessandro Severo del 222 d.C.), in cui si ribadisce il divieto di demolire edifici esistenti per trarne materiali edilizio da reimpiegare altrove. Il divieto di spoliazioni di edifici
} 
negli statuti locali sopra visti non pare ravvisarsi questa stessa esigenza; perlomeno, essi prevedono restrizioni per coloro che demolissero edifici senza autorizzazione preventiva e senza procedere a una ricostruzione degli stessi, indipendentemente dal fatto (non menzionato, ma comunque possibile) che tali speculazioni fossero legate al commercio di materiale edilizio. Negli statuti l'accento è posto in particolare sulla condizione necessaria per l'autorizzazione di un atto di demolizione: esso sarà infatti concesso solo se saranno date garanzie relative alla ricostruzione del fabbricato. Si ha quindi l'impressione che lo scopo delle norme non consistesse tanto, come in altri testi (ad esempio il senatus consultum "osidiano"), nell'evitare che si traesse materiale edilizio da edifici quanto piuttosto nel preservare il tessuto urbanistico, e quindi l'aspetto, della città. Ritorniamo allora al concetto di publicum decus sopra richiamato. ${ }^{36}$

Il concetto di estetica urbanistica sembra affermarsi soprattutto nel I secolo d.C.: esemplificativi di questa situazione sono i criteri che, secondo Tacito (Ann. 15.43), Nerone applicò nella ricostruzione dell'urbs devastata dall'incendio. Secondo Tacito, laddove la città, dopo il disastro gallico, era stata ricostruita nulla distinctione e con le case sparse qua e là, Nerone fece riprogettare l'intero tessuto urbanistico in base a regole ben precise, che, oltre a rispondere a esigenze di praticità, dovevano senza dubbio richiamarsi ad ideali estetici e di decoro. ${ }^{37}$

Anche il senatus consultum di età flavia menzionato da una costituzione di Alessandro Severo (C. 8.10.2), pur riferendosi in primo luogo al divieto di fare commercio di materiale edilizio di reimpiego, richiama il concetto di publicum decus, che potrebbe essere danneggiato in caso di spoliazione volontaria di edifici: ${ }^{38}$ negotiandi causa aedificia demoliri et marmora detrahere edicto divi Vespasiani et senatus consulto vetitum est. Ceterum de alia domo in aliam transferre quaedam licere exceptum est: sed nec dominis ita transferre licet, ut integris aedificiis depositis publicus deformetur adspectus.

Ancora Vespasiano, entro una serie di misure atte a migliorare il tessuto urbano di Roma, permise la costruzione di nuovi edifici in aree abbandonate e vuote, lasciate in queste condizioni dai proprietari (Suet. Vesp. 8.5): ${ }^{39}$ Deformis urbs veteribus incendiis ac ruinis erat; vacuas areas occupare et aedificare, si possessores cessarent, cuiusque permisit. Appare significativo il richiamo alle accuse mosse a Dione, e in particolare

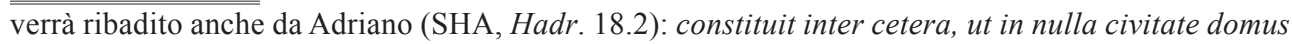
aliqua trasferendae ad aliam urbem ullius materiae causa dirueretur. Per la testimonianza del senatus consultum "aciliano", si veda NASTI 2017.

36 Malavé Osuna 2012.

37 Sulla legislazione, soprattutto tardoimperiale, che regolò il pubblico decoro anche degli edifici privati, si veda in particolare Robles Reyes 2001. Più in generale, Murga Gener 1976, Corbo 2019, p. 69. Si veda in particolare ProcCHI 2020, p. 165 per il tema della restitutio aedificii, «imposta dal potere imperiale con l'obiettivo di preservare l'integrità architettonica ed abitativa del tessuto urbano».

38 Sulla politica edilizia di Alessandro Severo si veda in particolare NASTI 2006, p. 160. Sul provvedimento di età flavia, ripreso da Alessandro Severo, si veda in particolare, oltre a BuONGIORNo 2010b, Malavé Osuna 2012, pp. 139-148, in part. pp. 140-141.

39 Su questo passo si veda in sintesi WARDLE 1996 
quelle che imputavano all'oratore di aver raso al suolo un'area di Prusa senza riuscire a costruirvi nulla di nuovo, lasciandole dunque, sostanzialmente deserte.

Dione, dunque, fu accusato di aver danneggiato la città di Prusa e il suo aspetto, ma non di fare commercio di materiali edilizi. ${ }^{40}$ Non è certo che a Prusa esistesse una legge sulla base della quale Dione potesse essere formalmente accusato. Noteremo, tuttavia, che, mentre a Roma, al di fuori del problema del commercio dei materiali di reimpiego, mancò per lungo tempo una regolamentazione che proibisse la demolizione degli edifici che non fosse seguita da una immediata fase di ricostruzione, a livello locale, in Italia come in provincia (si pensi alla legge di Taranto e alla Lex Ursonensis), tale obbligo fu espressamente previsto; ${ }^{41}$ similmente, per l'Urbs, Vespasiano pare essersi mosso in una direzione analoga, evitando spazi vuoti nei quartieri della città.

Le sopra menzionate epistole pliniane ci confermano, peraltro, come l'orizzonte delle accuse rivolte a Dione riguardasse soprattutto il decoro della città, danneggiato da Dione a causa di scelte di politica edilizia ritenute sbagliate: aree di Prusa rase al suolo cui non corrispose la ricostruzione, nei tempi previsti, dei fabbricati promessi. Plinio ci testimonia, come abbiamo visto, come le città della sua provincia, Prusa inclusa, fossero per così dire disseminate di opere incompiute, o per errori progettuali o per l'esaurirsi dei fondi a disposizione. Non stupisce, dunque, che anche Dione e il suo programma edilizio fossero andati incontro a problemi analoghi.

Tuttavia, mentre nel caso di demolizioni non autorizzate o non seguite da fasi di ricostruzioni sembrano emergere precisi divieti e provvedimenti, in questo caso non troviamo riferimenti a misure legislative che sanzionassero coloro che non terminassero i lavori promessi. ${ }^{42}$ Testimonianza di questa caratteristica è proprio il carteggio tra Plinio e Traiano, da cui emerge l'incertezza di Plinio a proposito dell'atteggiamento da prendere nei confronti di coloro che, per motivi vari e più o meno plausibili, lasciavano a metà o non finite le opere promesse alla comunità (e spesso finanziate anche da quest'ultima). In Bitinia non si tratta solo di opere demolite e non ricostruite, come previsto dagli statuti locali sopra visti; piuttosto, si tratta di opere non compiute, spesso sorte sulle macerie di precedenti fabbricati distrutti.

Si profila, per l'età traianea, un problema non di poco conto, vale a dire la mancata realizzazione di quanto promesso da privati cittadini nei confronti delle comunità di appartenenza, problema che sembra essere stato affrontato proprio dalla legislazione promossa da Traiano con una constitutio richiamata da Pomponio (D. 50.12.14): Si quis sui alienive honoris causa opus facturum se in aliqua civitate promiserit, ad perficiendum tam ipse quam heres eius ex constitutione divi Traiani obligatus est. Sed si

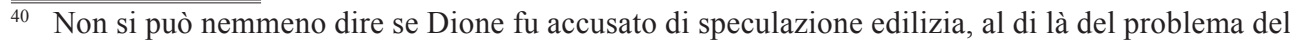
commercio illegale di materiale edilizio di reimpiego. A Roma, ad esempio, Crasso si sarebbe arricchito acquistando edifici in rovina per ricostruirne di nuovi (Plut. Crass. 2.4-5). Strabone (5.3.7) ci testimonia ulteriormente la diffusione di questa pratica.

41 Su questo aspetto si sofferma anche Marano 2013.

42 Su questo problema si veda ABERSON - HUFSCHMID 2012, che si concentrano, in particolare, sui casi in cui l'evergeta morisse prima della conclusione dell'opera. In generale, per il mondo greco, si veda PAPINI 2019.
} 
quis ob honorem opus facturum se civitate aliqua promiserit atque inchoaverit et priusquam perficeret, decesserit: heres eius extraneus quidem necesse habet aut perficere id aut partem quintam patrimonii relicti sibi ab eo, qui id opus facere instituerat, si ita mallet, civitati, in qua id opus fieri coeptum est, dare. Il princeps stabilisce che colui che, honoris causa (relativamente a sé o a un'altra persona), abbia promesso a una res publica la realizzazione di un opus sia obbligato a eseguire quanto promesso e iniziato (tale obbligo, peraltro, ricadrebbe anche sul suo erede). È vero che qui ci si richiama sulla questione specifica degli eredi e del comportamento che essi avrebbero dovuto assumere di fronte alla necessità di realizzare un'opera da altri promessa $\mathrm{e}$ soprattutto già iniziata, ${ }^{43} \mathrm{e}$ tuttavia non possiamo fare a meno di notare come la constitutio traianea cercasse, in generale, di obbligare a portare a termine quelle opere che, promesse, erano state interrotte (vuoi anche per la morte del pollicitator). Ritroviamo, insomma, nel provvedimento di Traiano echi di quella situazione che Plinio e Dione descrivono per la loro provincia proprio in età traianea, di cui, evidentemente, il princeps dovette in qualche modo occuparsi.

In questa medesima direzione si muoverà tempo dopo Antonino Pio, ${ }^{44}$ il quale, per primo e in forma esplicita, stabilì che le donazioni dei privati a favore della costruzione di nuovi edifici fossero da dirottare sulla manutenzione di quelli già esistenti, in modo da perseguire due vantaggi: da un lato, la manutenzione dei fabbricati esistenti, spesso lasciati in stato di abbandono, dall' altro, la diminuzione delle opere incompiute sparse per la città, in accordo a quel fenomeno stigmatizzato da Plinio il Giovane e ripreso da Dione di Prusa. Richiamandosi al concetto di tutela, da intendersi nel senso di "manutenzione" con specifico riferimento a disposizioni relative al comparto edilizio, un rescritto di Antonino Pio riportato da Callistrato prevede appunto che la pecunia relicta in opera nova possa essere impiegata più utilmente ed oculatamente (D. 50.10.7):45 Pecuniam quae in opera noua legata est, potius in tutelam eorum operum quae sunt conuertendam, quam ad inchoandum opus erogandam diuus Pius rescripsit; scilicet si satis operum ciuitas habeat et non facile ad reficienda ea pecunia inueniatur. Si è ritenuto che l'imperatore volesse porre un limite alle eccessive spese che riguardavano la costruzione di edifici, dirottando le risorse disponibili sulla manutenzione degli edifici già esistenti, distogliendole nel contempo dalla costruzione di nuove opere.

Per concludere, dunque, le accuse che furono mosse a Dione di Prusa non furono verosimilmente pretestuose, nella misura in cui riflettevano un cattivo costume che doveva essere assai diffuso (anche al di fuori di Prusa) e nel quale fu coinvolto anche Dione, forse per motivi da ricollegarsi alla vita politica locale ${ }^{46} \mathrm{o}$ forse perché davve-

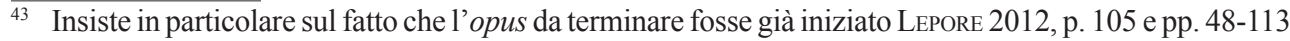
per un'approfondita analisi della constitutio traianea in questione.

44 Sui tentativi da parte dei primi imperatori della dinastia antonina di rimediare agli aspetti meno efficienti delle amministrazioni cittadine si veda in sintesi COMPARETTE 1906.

45 Su questo passo si veda Bosso 2006, SEGEnNI 2001, p. 357 e Russo c.d.s.

46 Sulle attività edilizie e di abbellimento che interessarono le città dell'Asia Minore tra I e II secolo d.C. e sull'ideologia a esse connesse, vd. in generale Pont 2010, p. 491. È verosimile che i problemi lamentati da Plinio e Dione siano da ricollegare proprio alla vivacità edilizia dell'inizio del II secolo
} 
ro, per motivi economici, pratici o di altra natura, l'oratore non fu in grado di portare a termine quanto promesso. Dalle accuse che gli furono rivolte emerge un'attenzione, non si può dire se strumentale o meno, al publicum decus della città: egli, infatti, non fu mai accusato di commercio illecito di materiali edilizi di reimpiego, quanto, piuttosto, di aver danneggiato l'aspetto della città.

A questo proposito, sottolineiamo una differenza fondamentale tra le accuse rivolte a Dione e la contemporanea legislazione edilizia sopra richiamata: la demolizione di un edificio appare infatti subordinata, in vario modo, alla semplice ricostruzione dello stesso edificio; il verbo usato è infatti restituere. Le demolizioni promosse da Dione, invece, oltre a non configurarsi come volgare speculazione per lucrare sulla rivendita di materiali edilizi, sono finalizzate non già alla ricostruzione degli stessi edifici (sia pure non in deterius), ma alla costruzione ex novo di edifici del tutto diversi, di uso pubblico ed espressamente concepiti per accrescere il publicum decus (un po' come previsto, molto prima, anche dallo statuto tarantino).

Proprio il pubblico decoro delle città dell'impero fu oggetto di legislazione per la prima volta sotto Vespasiano, forse a causa delle condizioni in cui oggettivamente versava Roma. E tuttavia, sarà solo con Antonino Pio, a mio avviso, che si procederà in maniera più sistematica a evitare che fabbricati incompiuti ledessero la venustas delle città dell'impero.

È verosimile, dunque, che spunti di questa legislazione fossero giunti anche in provincia e là declinati da parte delle amministrazioni delle diverse comunità. In effetti, l'insistenza di Dione sul fatto che il progetto avesse ricevuto più volte l'approvazione delle autorità locali (e non solo) sembrerebbe implicare proprio l'esistenza di disposizioni che vincolassero progetti di questo tipo a delibere degli organi cittadini; l'aver agito nel rispetto di questo avrebbe dovuto mettere Dione, nell'ottica dell'oratore stesso, al riparo da attacchi politici e strumentali.

In questo senso, insomma, le orazioni dionee costituiscono una preziosa quanto indiretta testimonianza della gestione degli edifici pubblici a livello locale e della parallela preoccupazione, in questo senso, sentita anche a Roma.

Federico Russo

Università degli Studi di Milano federico.russo@unimi.it

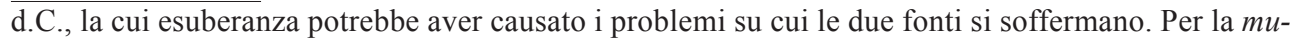
nificentia dei programmi edilizi delle province d'Asia Minore, vd. in sintesi BARRESI 2000; per la connessione tra quest'ultimi e lotta politica in Asia Minore (con attenzione anche alla documentazione archeologica) si veda RYAN 2018. Per il caso specifico della Bitinia si rimanda all'ampia trattazione di Fernoux 2004, pp. 329-360.
} 


\section{RIFERIMENTI BIBLIOGRAFICI}

Aberson - Hufschmid 2012 : M. Aberson, T. Hufschmid, Bâtiments publics inachéves: crises et solutions, in Gérer les territoires, les patrimoines et les crises, a cura di L. Lamoine, C. Berrendonner, M. Cébeillac-Gervasoni, Clermond Ferrand, Presses Universitaires BlaisePascal, 2012, pp. 247-260.

Амато 2014 : E. Amato, Traiani praeceptor: studi su biografia, cronologia e fortuna di Dione Crisostomo, Besançon, Presses Universitaires de Franche-Comté, 2014.

von Arnim 1898 : H. von Arnim, Leben und Werke des Dio von Prusa, Berlin, Weidmann, 1898.

Bekker-Nielsen 2008: T. Bekker-Nielsen, Urban Life and Local Politics in Roman Bithynia: The Smallworld of Dion Chrysostomos, Aarhus, Aarhus Universitetsforlag, 2008.

Billault 2014: A. Billault, L'image de Dion Chrysostome dans la correspondance de Pline le Jeune (Ep. 10, 81-82), in Autour de Pline le Jeune: en hommage à Nicole Méthy, a cura di E. Devillers, Bordeaux, Ausonius, 2014, pp. 238-244.

Bosso 2006 : F. Bosso, Della tutela più che della opera nova: la pratica delle costruzioni in Asia Minore nell'età di Antonino Pio, «Polis. Studi interdisciplinari sul mondo antico» 2 (2006), pp. 277-286.

Buongionno 2010a : P. Buongiorno, Senatus consulta Claudianis temporibus facta. Una palingenesi delle deliberazioni senatorie dell'età di Claudio (41-54 d.C.), Napoli, Edizioni Scientifiche Italiane, 2010.

Buongionno 2010b : P. Buongiorno, CIL X 1401 e il senatus consultum 'Osidiano', «Iura» 58 (2010), pp. 234-251.

Caballos Rufino 2018 : A. Caballos Rufino, El nuevo bronce de Osuna y la política colonizadora romana, Sevilla, Editorial Universidad de Sevilla, 2018.

Cappelletti 2011 : L. Cappelletti, Gli statuti di Banzi e Taranto nella Magna Graecia del I secolo d.C., Frankfurt am Main, Peter Lang, 2011.

CAPpelletti 2017 : L. Cappelletti, Norme per la tutela degli edifici negli statuti locali (secoli I a.C. - I d.C.), «Bullettino dell’Istituto di Diritto Romano» 111 (2017), pp. 53-74.

Comparette 1906 : T.L. Comparette, The Reorganization of the Municipal Administration under the Antonines, «The American Journal of Philology» 2 (1906), pp. 166-183.

CRAWFORD 1996 : M.H. Crawford, Roman Statutes, I-II, London, Institute of Classical Studies, 1996.

Corbo 2019 : C. Corbo, Diritto e decoro urbano in Roma antica, Napoli, Satura Editrice, 2019. 
Daguet-Gagey 2010 : A. Daguet-Gagey, Les opera publica dans la correspondance de Pline le Jeune avec Trajan, in L'étude des correspondances dans le monde romain de l'Antiquité classique à l'Antiquité tardive: permanences et mutations, a cura di J. Desmulliez, C. HoëtVan Cauwenberghe, J.-C. Jolivet, Lille, Éditions du Conseil Scientifique de l'Université de Lille, 2010, pp. 247-272.

Desideri 1978 : P. Desideri, Dione di Prusa, Messina-Firenze, G. D’Anna, 1978.

Desideri 2019 : P. Desideri, Ellenismo imperiale. Nuovi studi su Dione di Prusa, Pisa-Roma, Fabrizio Serra Editore, 2019.

Drolet 2004 : C. Drolet, La rivalité entre Nicomédie et Nicée, de l'époque d'Auguste à celle de Septime Sévère, «Cahiers des études anciennes» 41 (2004), pp. 72-91.

Dubouloz 2011 : J. Dubouloz, La propriété immobilière à Rome et en Italie. ${ }^{\mathrm{er}}-V^{\mathrm{e}}$ siècles, Roma, École Française de Rome, 2011.

Eck 1997: W. Eck, Der Evergetismus im Funktionszusammhang der kaiserzeitlichen Städte, in Actes $d u X^{\mathrm{e}}$ Congrès international d'épigraphie grecque et latine, atti del convegno (Nîmes 1992), a cura di M. Christol, O. Masson, Paris, Éditions de la Sorbonne, 1997, pp. 305-331.

ENGFER 2017 : K. Engfer, Die private Munifizenz der römischen Oberschicht in Mittel- und Süditalien. Eine Untersuchung lateinischer Inschriften unter dem Aspekt der Fürsorge, Wiesbaden, Otto Harrassowitz, 2017.

Fernoux 2004 : H.-L. Fernoux, Notables et élites des cités de Bithynie aux époques hellénistique et romaine: $I I I^{\mathrm{e}}$ siècle av. J.-C.-III' siècle ap. J.-C.: essai d'histoire sociale, Lyon, MOM Éditions, 2004.

Fernoux 2011 : H.-L. Fernoux, Le Demos et la cité: communautés et assemblées populaires en Asie mineure à l'époque impériale, Rennes, Presses Universitaires de Rennes, 2011.

GABBA 1994 : E. Gabba, Considerazioni politiche ed economiche sullo sviluppo urbano in Italia nei secoli II e I a.C., in Id., Italia romana, Como, New Press, 1994, pp. 105-118 (= in Hellenismus in Mittelitalien, atti del convegno [Göttingen 1974], a cura di P. Zanker, I-II, Göttingen, Vandenhoeck und Ruprecht, 1976, II, pp. 315-326).

GonzÁlez, Crawford 1986 : J. González, M.H. Crawford, The Lex Irnitana: A New Copy of the Flavian Municipal Law, «The Journal of Roman Studies» 76 (1986), pp. 147-243.

HARris 1992 : B.F. Harris, Dio of Prusa: A Survey of Recent Work, in Aufstieg und Niedergang der römischen Welt II.35.5, Berlin-New York, De Gruyter, 1992, pp. 3853-3881.

Heller 2006 : A. Heller, Les bêtises des Grecs. Conflits et rivalités entre cités d'Asie et de Bithynie à l'époque romaine (129 a.C.-235 p.C.), Bordeaux, Ausonius, 2006.

Johnston 1985 : D. Johnston, Munificence and Municipia: Bequests to Towns in Classical Roman Law, «The Journal of Roman Studies» 75 (1985), pp. 105-125. 
KLeIJWegt 1994 : M. Kleijwegt, Voluntarily, but under pressure. Voluntarity and constraint in Greek municipal politics, «Mnemosyne» 47 (1994), 64-78.

KoKkinia 2004 : C. Kokkinia, The philosopher and the emperor's words: Trajan, Flavius Archippus and Dio Chrysostom, «Historia» 53 (2004), pp. 490-500.

KoKKINIA 2006 : C. Kokkinia, The governor's boot and the city's politicians: Greek communities and Rome's representatives under the empire, in Herrschaftsstrukturen und Herrschaftspraxis: Konzepte, Prinzipien und Strategien der Administration im römischen Kaiserreich, atti del convegno (Zürich 2004), a cura di A. Kolb, Berlin, Akademie verlag, 2006, pp. 181-189.

LAFF 2007 : U. Laffi, Osservazioni sulla lex municipii Tarentini, in U. Laffi, Colonie e municipi nello stato romana, Roma, Edizioni di Storia e Letteratura, 2007, pp. 191-232 (= «Rendiconti lincei. Scienze morali, storiche e filologiche» 9 [2004], pp. 611-640).

Lamar Crosby 1946 : H. Lamar Crosby, Dio Chrysostom. Discourses 37-60, Cambridge Mass. London, Harvard University Press, 1946.

LAMBERTI 1993 : F. Lamberti, Tabulae Irnitanae. Municipalità e ius romanorum, Napoli, Jovene, 1993.

LEwIN 1995 : A. Lewin, Assemblee popolari e lotta politica nelle città dell'impero romano, Firenze, Editrice La Giuntina, 1995.

Malavé Osuna 2012 : B. Malavé Osuna, El esplendor de las ciudades: “decus publicum” y estética urbana, in Fvndamenta ivris. Terminología, principios e "interpretatio", a cura di P. Resina Sola, Almería, Universidad de Almería, 2012, pp. 139-148.

MARANo 2013 : Y.A. Marano, 'Roma non è stata (de)costruita in un giorno'. Fonti giuridiche e reimpiego in età romana (I secolo a.C. - VI secolo d.C.), «Lanx» 16 (2013), pp. 1-54.

MÉTHy 1994: N. Méthy, Dion Chrysostome et la domination romaine, «L'Antiquité Classique» 63 (1994), pp. 173-192.

Milazzo 1993 : F. Milazzo, La realizzazione delle opere pubbliche in Roma arcaica e repubblicana. Munera e ultro tributa, Napoli, Edizioni Scientifiche Italiane, 1993.

MROzeK 1987 : A. Mrozek, Les distributions d'argent et de nourriture dans les villes italiennes du Haut-Empire romain, Bruxelles, Latomus Revue d'Etudes Latines, 1987.

Murga Gener 1976: J.L. Murga Gener, Protección a la estética en la legislación urbanística del Alto Imperio, Sevilla, Publicaciones de la Universidad de Sevilla, 1976.

Nasti 2006 : F. Nasti, L'attività normativa di Severo Alessandro. Politica di governo, riforme amministrative e giudiziarie, Napoli, Satura Editrice, 2006.

NASti 2017 : F. Nasti, Mutare, detrahere, transferre: considerazioni sui senatusconsulta Osidiano, Aciliano e l'ad Sabinum di Ulpiano, «Studia et Documenta Historiae et Iuris» 83 (2017), pp. 591-602. 
PAPINI 2019 : M. Papini, «Pendono interrotte le opere». Antichi monumenti incompiuti nel mondo greco, Roma, Sapienza Editrice, 2019.

PhiLips 1973 : E.J. Philips, The Roman Law on the Demolition of Buildings, «Latomus» 32 (1973), pp. 86-95.

Pont 2010 : A.-V. Pont, Orner la cité: enjeux culturels et politiques du paysage urbain dans l'Asie gréco-romaine, Pessac, Ausonius Éditions, 2010.

Procchi 2001a : F. Procchi, La tutela urbanistica: un problema non nuovo. Considerazioni a margine del SC. Hosidianum, in Scritti in onore di Antonio Cristiani. Omaggio della Facoltà di Giurisprudenza dell'Università di Pisa, a cura di G. De Francesco, Torino, Giappichelli, 2001, pp. 659-676.

Procchi 2001b : F. Procchi, “Si quis negotiandi caussa emisset quod aedificium...”. Prime considerazioni su intenti negoziali e "speculazioni edilizie” nel principato, «Labeo» 47 (2001), pp. 411-438.

Procchi 2020 : F. Procchi, La conservazione delle abitazioni nel contesto urbano: prime osservazioni sul pubblico intervento "ob restitutionem aedifici" in età imperiale, in La tutela dei "beni culturali” nell'esperienza giuridica romana, a cura di F. Fasolino, Milano, Wolters Kluwer, 2020, pp. 151-165.

RAINER 1987 : J.M. Rainer, Bau- und nachbarrechtliche Bestimmungen im klassischen römischen Recht, Graz, Leykam, 1987.

RAINER 1991 : J.M. Rainer, Zu den Abbruchstimmungen in den Städterechten, «Zeitschrift der Savigny Stiftung» 108 (1991), pp. 325-329.

Robles Reyes 2001 : J.R. Robles Reyes, De la protección de los edificios privados. Estudio comparado de los textos municipales hispanos del siglo I, el código de Justiniano y las siete partidas, «Anales de derecho (Universidad de Murcia)» 19 (2001), pp. 181-193.

Russo c.d.s. : F. Russo, La tutela degli edifici nelle città dell'impero romano in età antonina. Considerazioni in margine a D. 50.10.7, «Latomus» c.d.s.

Ryan 2018 : G. Ryan, Building Order: Unified Cityscapes and Elite Collaboration in Roman Asia Minor, «ClAnt» 37 (2018), 151-185.

Salmeri 1980 : G. Salmeri, Per una biografia di Dione di Prusa. Politica ed economia nella Bitinia imperiale (I-II sec. d. C.), «Siculorum Gymnasium» 33 (1980), pp. 671-715.

SAlmeri 1982 : G. Salmeri, La politica e il potere. Saggio su Dione di Prusa, Catania, Facoltà di Lettere e Filosofia, Università di Catania, 1982.

SAlmeri 1999 : G. Salmeri, La vita politica in Asia Minore sotto l'impero romano nei discorsi di Dione di Prusa, in Lancia, diadema e porpora. Il re e la regalità ellenistica, "Studi Ellenistici» 12 a cura di B. Virgilio (1999), pp. 211-267. 
SARGENTI 1984 : M. Sargenti, La disciplina urbanistica a Roma nella normativa di età tardorepubblicana e imperiale, in La città antica come fatto di cultura, atti del convegno (ComoBellagio 1979), a cura di M.A. Levi, A. Biscardi, Como, Società Archeologica Comense, 1984, pp. 265-284.

SARgenti 1984a : M. Sargenti, Due senatoconsulti - Politica edilizia nel primo secolo dell'impero e tecnica normativa, in Studi in onore di Cesare Sanfilippo, I-VIII, Milano, Giuffrè, 1984, V, pp. 637-656.

Schmitz 1996 : T. A. Schmitz, Trajan und Dion von Prusa: zu Philostrat, Vit. Soph. 1, 7 (488), «RhM» 139 (1996), pp. 315-319.

Segenni 2001 : S. Segenni, Antonino Pio e le città dell'Italia. Riflessioni su H.A., v. Pii, 8, 4, «Athenaeum» 89 (2001), pp. 355-405.

Sheppard 1984 : A.R.R. Sheppard, Dio Chrysostom, the Bithynian years, «AC» 53 (1984), pp. $157-173$.

Sherwin White 1966 : A.N. Sherwin White, The Letters of Pliny: A Historical and Social Commentary, Oxford, Oxford University Press, 1966.

Swain 2000 : S. Swain, Dio Chrysostom. Politics, Letters, and Philosophy, Oxford, Oxford University Press, 2000.

Tномаs 1998 : Y. Thomas, Les ornements, la cité, le patrimoine, in Images romaines, Atti del convegno (Parigi 1996), a cura di C. Auvray-Assayas, Paris, Éditions Rue d'Ulm 1998, pp. 263-284.

Trisoglio 1972 : F. Trisoglio, Le idee politiche di Plinio il Giovane e di Dione Crisostomo, «Il Pensiero politico» 5 (1972), pp. 3-43.

Vielmetti 1941 : C. Vielmetti, I discorsi bitinici di Dione Crisostomo, «Studi italiani di filología classica» 18 (1941), pp. 89-108.

Wardle 1996 : D. Wardle, Vespasian, Helvidius Priscus and the Restoration of the Capitol, «Historia» 45 (1996), pp. 208-222.

WINTER 1996 : E. Winter, Staatliche Baupolitik und Baufürsorge in den römischen Provinzen des kaiserzeitlichen Kleinasien, Bonn, Dr. Rudolf Habelt, 1996.

ZaCCARIA RugGiU 1990 : A. Zaccaria Ruggiu, L'intervento pubblico nella regolamentazione dello spazio privato. Problemi giuridici, «RdA» 14 (1990), pp. 77-94.

Zuiderhoek 2009 : A. Zuiderhoek, The Politics of Munificence in the Roman Empire. Citizens, Elites and Benefactors in Asia Minor, Cambridge, Cambridge University Press, 2009. 